\title{
How Annealing and Charge Scavengers Affect Visible Emission from ZnO Nanocrystals
}

\author{
Matthew E. Reish, ${ }^{\dagger}$ Zhen Zhang, ${ }^{\dagger}$ Shiliang Ma, ${ }^{\dagger}$ Ian Harrison, ${ }^{\dagger}$ and Henry O. Everitt ${ }^{*,+, \S}$ \\ ${ }^{\dagger}$ Department of Chemistry, University of Virginia, Charlottesville, Virginia 22904-4319, United States \\ ${ }^{\ddagger}$ U.S. Army Aviation \& Missile RD\&E Center, Redstone Arsenal, Alabama 35898, United States \\ ${ }^{\S}$ Department of Physics, Duke University, Durham, North Carolina 27708, United States
}

\section{Supporting Information}

ABSTRACT: Simultaneous transmission infrared (IR) absorption and photoluminescence $(\mathrm{PL})$ spectroscopies are used to reveal the correlation of free electron and defect densities during the stepwise annealing of $\mathrm{ZnO}$ nanocrystals in high vacuum. For increasing annealing temperatures between 700 and $1000 \mathrm{~K}$, the free electron density increases with a negligible increase in PL yield. With increased annealing temperature above $1000 \mathrm{~K}$, the free electron density decreases and the PL yield increases in inverse proportion. Accompanying the free electron loss are indications of increased bound charges and changes in the multiphonon bands in the

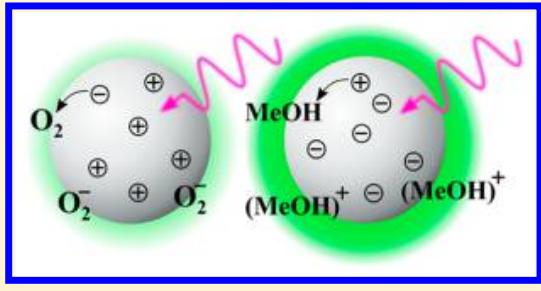
infrared spectrum, which collectively suggest that structural change and defect formation accompanies the loss of free electrons and the increase in PL. Exposure of the previously annealed sample to electron $\left(\mathrm{O}_{2}\right)$ and hole $(\mathrm{MeOH})$ scavengers shows that the buildup of holes quenches visible emission, while additional electrons have a marginal effect on the PL yield. Given that certain neutral donors bind excitons and facilitate energy transfer to visible emitting sites, the buildup of free holes appears to quench PL intensity by ionizing those neutral donors.

\section{INTRODUCTION}

The complex vacancy- and interstitial-related visible photoluminescence in $\mathrm{ZnO}$ has been actively explored for practical device applications such as low-cost, low-toxicity, visible wavelength phosphors. ${ }^{1,2}$ Although near-UV band edge emission is dominant in highly ordered crystals, annealing or other defect creation methods lead to efficient green emission $(\sim 2.4 \mathrm{eV}){ }^{3}$ Previously, the visible emission was attributed to substitution of copper for zinc $^{4-6}\left(\mathrm{Cu}_{\mathrm{Zn}}\right)$, oxygen, ${ }^{7-12}$ or zinc $^{13-16}$ vacancies $\left(\mathrm{V}_{\mathrm{O}}, \mathrm{V}_{\mathrm{Zn}}\right)$, oxygen ${ }^{17}$ or zinc interstitials ${ }^{18,19}$ $\left(\mathrm{O}_{\mathrm{i}}, \mathrm{Zn}_{\mathrm{i}}\right)$, or antisites of oxygen and $\operatorname{zinc}^{20}\left(\mathrm{O}_{\mathrm{Zn}}\right)$. Initially copper substitution was considered the dominant mechanism, often from unintentional doping. ${ }^{5,6}$ Further studies revealed that intrinsic defects could create centers for visible PL. Early evidence suggested that the creation of singly ionized oxygen vacancies $\left(\mathrm{V}_{\mathrm{O}}^{-}\right)$and an increase in free charges correlated with an increase in visible PL. ${ }^{7-9}$ However, the EPR assignment of $g$ $=1.956$ for the singly ionized oxygen vacancy was questioned, and the method used to detect free charges by changes in band edge absorption may not be reliable. ${ }^{1,11,12}$ Later, optically detected magnetic resonance measurements also suggested that oxygen vacancies were the origin of PL increase, but oxygen vacancies were assigned to a different EPR resonance, and an anticorrelation between the $g=1.956$ signal and PL intensity was found. ${ }^{11}$ Recent studies in which $\mathrm{ZnO}$ samples were annealed in both oxygen- and zinc-rich environments have established that both $\mathrm{V}_{\mathrm{O}}$ and $\mathrm{V}_{\mathrm{Zn}}$ lead to increased defect emission, but the origin of this emission is still debated. ${ }^{17,21-23}$

In this work we demonstrate that simultaneous IR absorption and visible emission spectroscopies can be used to investigate the correlation of carrier density and PL in high vacuum annealed $\mathrm{ZnO}$ nanocrystals. Free carrier densities have previously been shown to correlate with both increases ${ }^{7-9}$ and decreases ${ }^{17}$ in PL efficiency. In $\mathrm{ZnO}$ and other reducible metal oxides, it is well-known that the introduction of electrons into states at or near the conduction band generates absorption in the IR, which increases exponentially toward lower wavenumber. $^{24-28}$ In this study we use this characteristic IR absorption feature as a proxy for free carrier density to establish the role of free electrons in $\mathrm{ZnO}$ visible PL. IR spectra also include other information that can be used to gain an understanding of annealing related changes. In the 700-1100 $\mathrm{cm}^{-1}$ range, phonon overtone and combination bands are observed, and changes to these bands indicate lattice distortion. $^{29-31}$ Additionally, the appearance of a broad absorbance in the higher wavenumber range $\left(>2000 \mathrm{~cm}^{-1}\right)$ at high temperatures is an indication of an increase in neutral donor concentration. ${ }^{32-34}$

PL experiments on the annealed samples, including exposure to hole and electron scavengers, indicate that the buildup of free holes quenches visible emission. This supports recent findings which showed that visible $\mathrm{PL}$ in annealed $\mathrm{ZnO}$ is mediated by neutral donor sites which bind excitons. ${ }^{35}$ While a precise mechanism has not been elucidated, it was found that the commonly labeled $\mathrm{I}_{3 \mathrm{a}}$ neutral donor bound exciton (DBE) facilitates energy transfer to deep gap states that emit in the

Received: December 10, 2015

Revised: February 10, 2016 
visible region as shown in Figure 1. In this context we suggest that quenching of visible PL is the result of free holes ionizing the neutral donors responsible for mediating visible emission.

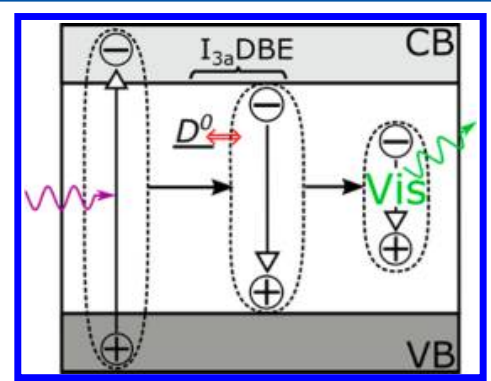

Figure 1. Schematic diagram illustrating how neutral DBE's facilitate visible emission.

\section{EXPERIMENTAL SECTION}

Nanocrystalline $\mathrm{ZnO}$ (Alfa Aesar 99.99\%, 300 nm mean particle size) was pressed into a tungsten grid to create a supported pellet with a diameter of $7 \mathrm{~mm}$ and thickness of 0.05 $\mathrm{mm}$. During annealing the nanoparticles are expected to agglomerate into particles of around $2 \mu \mathrm{m}$ based on previous experiments using similar annealing conditions. ${ }^{22,35}$ As described previously, the tungsten grid was clamped onto two copper bars attached to the copper wires of a vacuum feedthrough at the base of a dewar used to hold liquid nitrogen or acetone/dry ice for cooling. ${ }^{36}$ Heating was supplied to the sample by passing a current through the tungsten grid. The temperature was sensed by a K-type thermocouple spot-welded to the grid, and temperature was controlled by the a home-built proportional-integral-derivative (PID) controller, which maintained the temperature within $1 \mathrm{~K}$ and was programmed to the ramp the temperature at $4 \mathrm{~K} / \mathrm{s}$ during temperature changes. ${ }^{37}$ The sample was placed into a vacuum system with a base pressure of $7 \times 10^{-10}$ Torr and heated for $2 \mathrm{~h}$ at $700 \mathrm{~K}$ under 2 Torr of oxygen to remove any water, hydroxyl, or organic contaminants, and the disappearance of these contaminants was monitored in the IR with the full IR spectrum of the cleaned sample shown in Supporting Information S1. The sample holder was positioned at $45^{\circ}$ to both IR and UV excitation sources so that transmission IR and photoluminescence spectra could be collected simultaneously. Differentially pumped $\mathrm{KBr}$ windows in the vacuum cell allowed the transmission of IR light, and IR spectra were collected using a PerkinElmer Spectrum 100 with a liquid nitrogen cooled MCT detector. IR spectra were all collected with 128 repeated scans with a resolution of $4 \mathrm{~cm}^{-1}$. A glass window allowed excitation and collection of PL light using two optical fibers with collimating lenses at the sampling end. The PL excitation source was a 365 $\mathrm{nm}$ LED (LED Engin) whose emission was passed through a $10 \mathrm{~nm}$ band-pass filter to limit the excitation bandwidth. Emitted light was analyzed using a Silver Nova dispersive spectrograph (Stellar Net) with a $405 \mathrm{~nm}$ long pass filter to remove excitation light. $250 \mathrm{PL}$ exposures of $250 \mathrm{~ms}$ were averaged for each scan.

\section{RESULTS AND DISCUSSION}

Sequential Annealing of $\mathrm{ZnO}$. Vacuum annealing of $\mathrm{ZnO}$ was performed in $20 \mathrm{~K}$ steps from 700 to $1240 \mathrm{~K}$, with the temperature maintained at each step for $10 \mathrm{~min}$, and then returned to $300 \mathrm{~K}$ where simultaneous IR and PL spectra were collected. Figure 2A shows the full PL spectra after annealing at several temperatures. The integrated intensity $(400-700 \mathrm{~nm})$
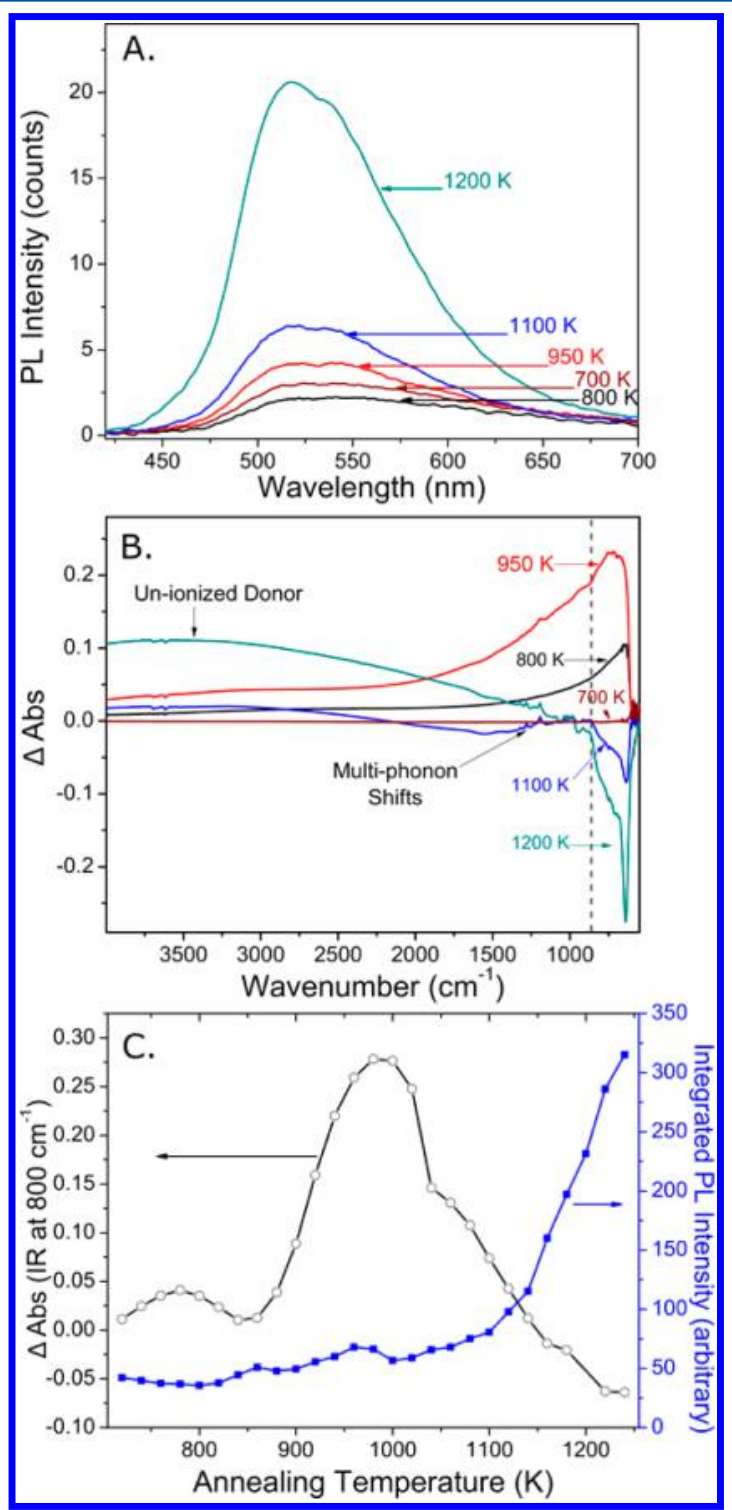

Figure 2. (A) PL spectra recorded at $300 \mathrm{~K}$ after annealing to 700 , $800,950,1100$, and $1200 \mathrm{~K}$. (B) IR absorbance difference spectra, relative to a $300 \mathrm{~K}$ reference spectrum, recorded simultaneously with the PL spectra. (C) $\Delta \mathrm{Abs}$ recorded at $800 \mathrm{~cm}^{-1}$ (vertical dashed line in panel B) and integrated PL intensity recorded after annealing in 20 $\mathrm{K}$ increments between 700 and $1240 \mathrm{~K}$.

of the PL spectra after each annealing cycle is plotted in Figure 2C. Figure 2B displays IR absorbance difference spectra: IR spectra subtracted from a reference recorded at $300 \mathrm{~K}$ before the annealing experiment began but after cleaning the sample in oxygen at $700 \mathrm{~K}$.

After annealing steps from 700 to $1000 \mathrm{~K}$ the PL intensity is relatively unchanged, at first falling slowly, which may be explained by the initial healing of defects, and then rising slightly. In the same temperature range, a strong increase in IR absorption is observed. The increased absorption rises with a power law dependence toward low wavenumber and is assigned to an increase in the free electron density based on this band shape. ${ }^{32,38}$ The absorbance can be characterized by a simple 
electromagnetic model that takes into account the effective mass of the electron using ${ }^{38}$

$$
\Delta \mathrm{Abs}=S(\tilde{\nu})^{-p}
$$

where $S$ is a scaling factor, $\tilde{v}$ is the frequency in wavenumbers, and the exponent $p$ is characteristic of the effective mass of the electron. An exponent of 1.75 suggests scattering from acoustic phonons in agreement with other polar metal oxide semiconductors with low trap densities. ${ }^{38,39}$ The data were fit using eq 1 between 2000 and $640 \mathrm{~cm}^{-1}$ and extrapolated to larger wavenumbers (Figure 3), revealing a second peak around 2800 $\mathrm{cm}^{-1}$ which has previously been identified as an indication of neutral donors. ${ }^{32-34}$

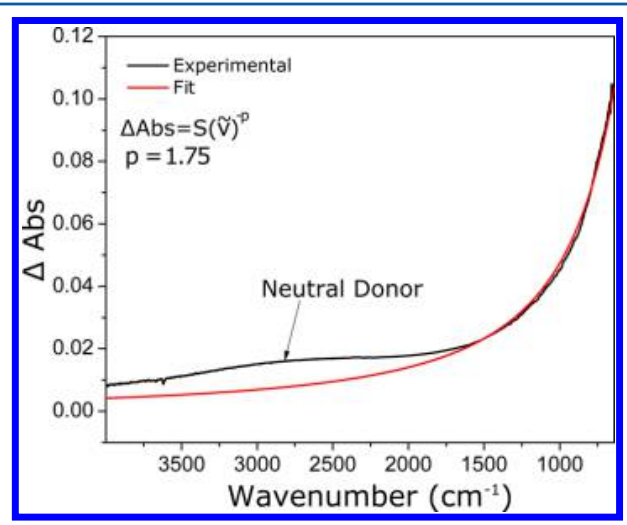

Figure 3. Fit of power-law increase of differential absorbance $\Delta \mathrm{Abs}$ toward low wavenumbers using eq 1 . The sample was annealed to 800 $\mathrm{K}$ and then cooled to $300 \mathrm{~K}$ to acquire the spectrum. Fitting was performed between 2000 and $640 \mathrm{~cm}^{-1}$ and extrapolated to reveal the effects of neutral donors that are also formed during annealing.

A similar free electron signal is noted for $\mathrm{ZnO}$ and several other reducible metal oxides. ${ }^{24-26,32,40}$ An increase in the free electron signal in the IR and in conductivity measurements during thermal annealing is often attributed to the loss of neutral oxygen, creating ionized vacancies or $\mathrm{Zn}$ interstitials. $^{41-43}$ Some theoretical studies with experimental corroboration suggest that oxygen vacancies in $\mathrm{ZnO}$ should generate deep levels around $0.7-1.0 \mathrm{eV}$ below the bottom of conduction band and should not be responsible for free carrier generation. $^{44,45}$ Though the source of shallow donors remains unresolved in the literature, in our high vacuum annealing experiments it is unlikely that a significant number of extrinsic donors are incorporated. We therefore suggest that shallow $V_{O}$ donors are responsible for increased free electron concentration, but we cannot completely rule out unintentional doping with contaminants such as hydrogen. In any case, the key result is that the increase in the free electron signal corresponds to only a negligible increase in PL. ${ }^{7-9}$

At annealing temperatures above $1000 \mathrm{~K}$, the free electron signal begins to decrease sharply, and a simultaneous increase in the PL intensity is observed. As seen in Figure 4, there is a clear inverse correlation between free electron density and PL intensity. The PL band shape during annealing slightly blueshifts as the number of oxygen vacancies increases, as shown in the Supporting Information (S2). ${ }^{22}$

The decrease of the free electron signal may be caused by damage to the $\mathrm{Zn}$ or $\mathrm{O}$ sublattice that generates an increased number of deep defect sites for electrons to reside, resulting in electron localization, reduced IR absorption, and an increased

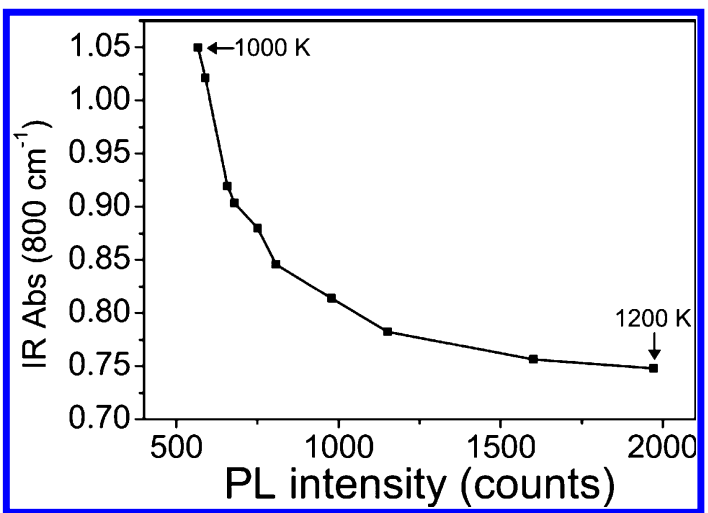

Figure 4. Inverse correlation of the integrated PL intensity and free electron IR absorbance at $800 \mathrm{~cm}^{-1}$ during successive annealing cyles.

number of sites that can produce visible emission. ${ }^{17}$ Additional indications for lattice damage and deep defect sites are observed in the IR spectra with new positive peaks appearing in the multiphonon region of the IR spectra at annealing temperatures near $950 \mathrm{~K}$, as shown in Figure 2B (for reference, compare with Figure S1 which shows the multiphonon peaks before annealing). These features are likely to be associated with changes in the $\mathrm{ZnO}$ lattice. The combination of two effects-new peaks appearing around the same annealing temperatures as the free electron signal begins to decreasesuggests that damage to the lattice may create defect sites that are occupied by previously free electrons. Evidence for additional deep gap sites is also observed in the IR spectra with a broad increase in absorbance toward higher wavenumbers at higher annealing temperatures. Specifically, a broad peak absorbance in the $2000-4000 \mathrm{~cm}^{-1}$ range increases at higher temperatures $(>1000 \mathrm{~K}$, Figure $2 \mathrm{~B})$, which has previously been associated with the presence of un-ionized donors in $\mathrm{ZnO}^{32-34}$

Dosing of Hole and Electron Scavengers. Shallow penetration depths of UV light in $\mathrm{ZnO}$ and the high surface area of microcrystalline particles make the properties of the surface important for understanding emission. The transfer and extraction of holes and electrons at the surface can be controlled by choosing adsorbates that can be oxidized or reduced at potentials of the free and trapped holes and electrons in $\mathrm{ZnO}$. In the hole and electron scavenger experiments, the sample previously annealed up to $1240 \mathrm{~K}$ was cooled to $100 \mathrm{~K}$ or held at $300 \mathrm{~K}$, and simultaneous PL and IR scans were taken. The lower temperature experiment shows enhanced charge trapping and a greater response to charge scavengers caused by lower charge mobility. Additionally, $\mathrm{MeOH}$ is physisorbed onto $\mathrm{UV}$-exposed $\mathrm{ZnO}$ at $100 \mathrm{~K}$, while at $300 \mathrm{~K}$ a chemisorbed layer of decomposed methanol is formed (Supporting Information S3). We present the $100 \mathrm{~K}$ experimental results here and report the $300 \mathrm{~K}$ results in the Supporting Information (S4). Importantly, the direction of IR absorption and PL intensity change are the same at both temperatures, and only the magnitude is reduced at $300 \mathrm{~K}$, so the same mechanisms operate at both temperatures. The integrated visible PL intensity $(400-700 \mathrm{~nm})$ and the IR absorbance at $1760 \mathrm{~cm}^{-1}$ during UV and gas exposure at $100 \mathrm{~K}$ are shown in Figure 5. The choice of $1760 \mathrm{~cm}^{-1}$ was made to avoid vibrational absorbance from methanol (full spectra shown in Supporting Information S5). 


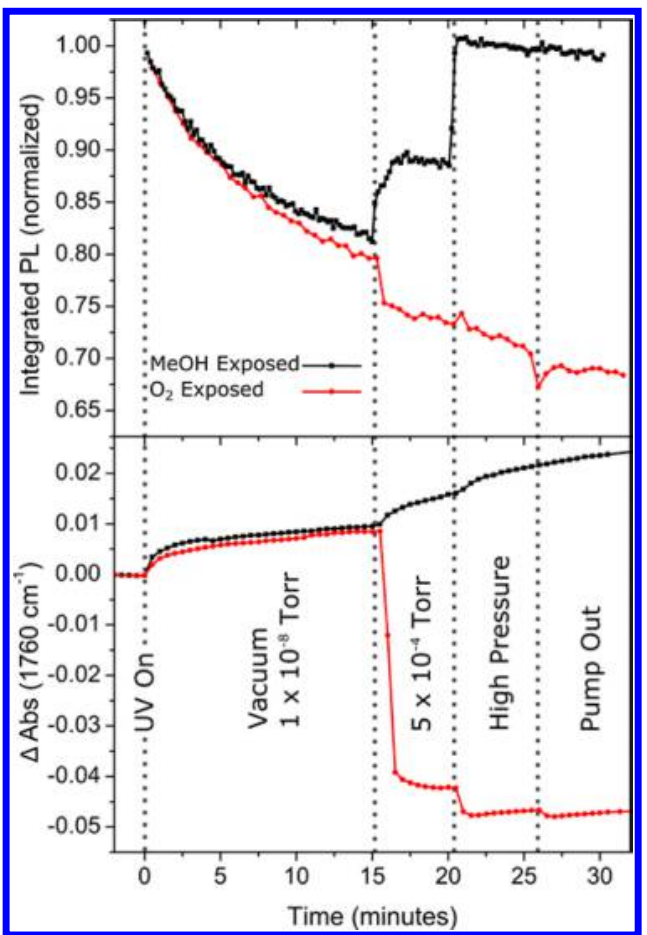

Figure 5. Top: integrated PL intensity during UV and gas exposure normalized to the first scan. Bottom: change in absorbance at 1760 $\mathrm{cm}^{-1}$ during UV and gas exposure. In both graphs, the black line represents the $\mathrm{MeOH}$-exposed sample and red line represents the $\mathrm{O}_{2-}$ exposed sample. The vertical dashed lines show timing of labeled events.

In vacuum the PL intensity decreases exponentially with a time constant of approximately $400 \mathrm{~s}$ and approaches a final value just below $80 \%$ of the initial PL intensity (see Supporting Information S6). At the same time the free electron signal in the IR increases and saturates around 0.01 absorbance units at $1760 \mathrm{~cm}^{-1}$. The loss of visible PL intensity during UV exposure is likely caused by a buildup of trapped charges that cause a shift to UV emission or nonradiative decay. ${ }^{46-48}$

To understand how the buildup of holes and electrons affects the visible emission in the stepwise-annealed samples of $\mathrm{ZnO}$, we exposed the sample to electron $\left(\mathrm{O}_{2}\right)$ and hole $(\mathrm{MeOH})$ scavengers and observed the effect of exposure on both the IR absorption and PL yield. For both gases the same conditions were used, with a background pressure of $5 \times 10^{-4}$ Torr supplied to the chamber for a period of $5 \mathrm{~min}$, indicated by the second vertical dashed line in Figure 5. Upon exposure to oxygen, a small decrease in the PL intensity is noted, and a relatively large IR absorbance decrease of 0.04 is observed in the free electron region. This result confirms that the IR absorption signal derives from the free electron density. During exposure to methanol, the PL intensity rises sharply along with a simultaneous but modest increase in the free electron IR signal.

Next, the full pressure in the gas line was released to the chamber, represented by the third dashed line in Figure 5. This gave a pressure of 2.4 Torr for $\mathrm{O}_{2}$ and 0.2 Torr for $\mathrm{MeOH}$, although the latter pressure decreased quickly to less than 1 mTorr as $\mathrm{MeOH}$ cryo-trapped onto the liquid nitrogen dewar used to cool the sample. After the high pressure dose of $\mathrm{O}_{2}$, an additional decrease of the free electron signal was noted, but no significant effect was noted in the PL spectrum. In the case of $\mathrm{MeOH}$, the high pressure dose again sharply increased the PL intensity, reaching an intensity higher than the initial value, while the free electron IR signal increased modestly. In all cases, the band shape of visible PL was unaffected during changes in intensity caused by UV exposure or scavenger dosing, suggesting the emitting state is unchanged (Supporting Information S7).

Previous research on freshly prepared, unannealed nanoparticles in suspensions of alkoxy solvent have exhibited the opposite effect to that observed here. ${ }^{46,49,50}$ In those reports, the efficiency of visible PL from alkoxy suspended nanoparticles was reduced during exposure to UV light as the solution was deoxygenated, and PL was restored when oxygen was admitted to the solution. To explain why visible PL is quenched by hole scavengers in these unannealed nanoparticles, it was proposed that deeply trapped holes mediate visible emission by recombining with donor electrons in shallow traps. However, in our annealed micron-sized powders, visible PL increases when exposed to hole scavengers. To understand this apparent contradiction, we note that recent work using vacuum annealed powders has shown that donor bound excitons (DBEs) are important for the transfer of energy from band gap states to the deep-trapped states responsible for visible PL. ${ }^{35}$ Using measured PL frequencies and deduced binding energies, two DBEs that facilitated energy transfer to the deep trapped state

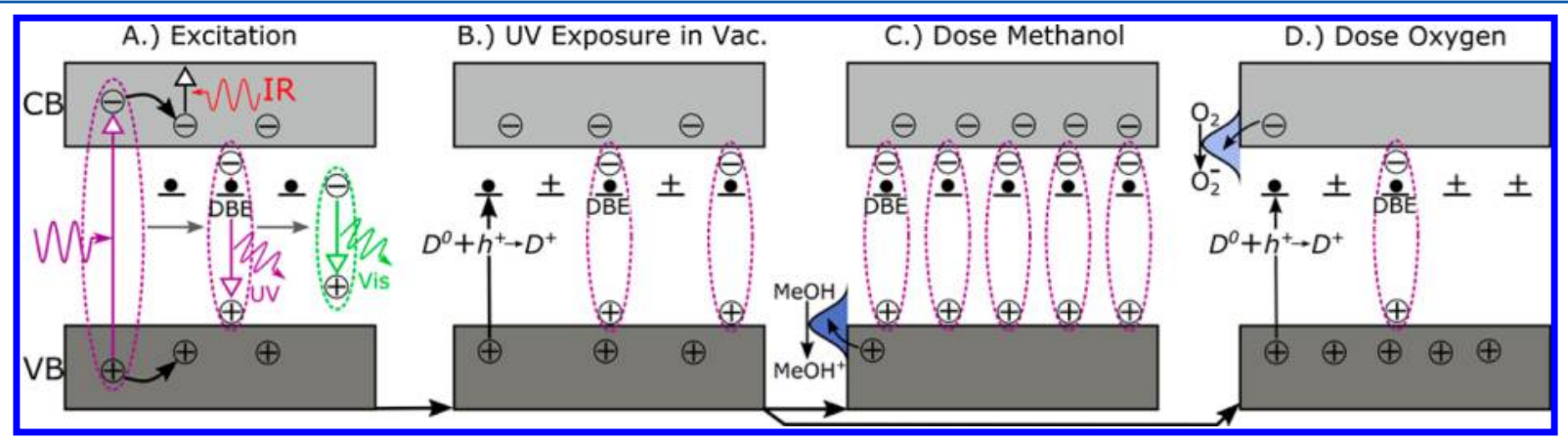

Figure 6. Schematic of DBE mediated visible PL and hole quenching in annealed $\mathrm{ZnO}$. (A) UV excitation of $\mathrm{ZnO}$ creates an exciton (dotted lines) which can separate into free charges, with free electrons observed by IR spectroscopy, or bind to a donor site to create a donor bound exciton (DBE). The DBE can then transfer energy to a visible emitting state. The horizontal lines in the bandgap represent neutral $(\bullet)$ and ionized $(+)$ donors. (B) In a vacuum at $100 \mathrm{~K}$, the buildup of free holes during UV exposure ionizes the neutral donors responsible for facilitating exciton binding and visible emission. (C) Exposure to methanol removes free holes, increases the free electron concentration, and regenerates neutral DBEs which facilitate visible emission. (D) Exposure to oxygen removes free electrons and increases the amount of free holes to ionize neutral donors. 
were identified, commonly labeled $\mathrm{I}_{9}$ and $\mathrm{I}_{3 a}{ }^{35,51,52}$ both of which are associated with neutral donors.

We therefore suggest that the visible PL intensity decrease during UV exposure is a result of free holes ionizing the $\mathrm{I}_{9}$ and $\mathrm{I}_{3 \mathrm{a}}$ neutral donors at sites created by the annealing process, thereby eliminating the efficient path previously identified for the generation of visible emission. This process is shown schematically in Figure 6A-D. Part A shows above band gap excitation and visible PL mediated by the neutral DBE. Part B shows the suggested mechanism for the loss of visible PL during UV exposure: reaction of photogenerated free holes with neutral donors. In part $\mathrm{C}$, the recovery of visible $\mathrm{PL}$ and increase in free electron concentration during $\mathrm{MeOH}$ exposure is illustrated, and in part $\mathrm{D}$ the further loss of visible $\mathrm{PL}$ and the loss of free electron density during $\mathrm{O}_{2}$ exposure is shown.

Evidence for this model comes from both annealing and scavenger dosing experiments. From the scavenger dosing data, outlined in Figure 5 and shown schematically in Figure 6C,D, it is seen that visible PL is very sensitive to the hole concentration, which is noted by the sharp increase of PL during the dosing of hole scavenging $\mathrm{MeOH}$, accompanied by a minor increase in the free electron signal in the IR. Conversely, the $\mathrm{PL}$ is relatively insensitive to the concentration of free electrons, as seen by the minor decrease of PL signal during $\mathrm{O}_{2}$ dosing accompanied by a large loss of the free electron signal. During annealing above $1000 \mathrm{~K}$ we note that increased PL intensity accompanies a loss of the free electron signal in the IR. This is likely caused by neutral donor site creation where previously free electrons were captured. ${ }^{53}$ Further evidence for the creation of neutral donors comes from the increased absorbance in the higher wavenumber region, as seen in Figures $2 \mathrm{~b}$ and 3 , which has been previously associated with the creation of neutral donors. $^{32-34}$

\section{CONCLUSIONS}

Simultaneous IR and PL spectroscopies are used to explore the role free carriers and neutral donors play in producing visible emission during stepwise annealing of $\mathrm{ZnO}$ nanocrystals. High temperature $(>1000 \mathrm{~K})$ annealing studies indicate that increases in visible PL emission intensity correlate with a decrease in free electron concentration. Exposure of the annealed sample to a hole scavenger enhances PL lost during UV irradiation in vacuum, suggesting that a buildup of free holes quenches fluorescence. Previous experiments showed that neutral DBEs facilitate energy transfer to visible light-emitting deep-trap states in annealed powders; therefore, we hypothesize that this quenching is an indication that neutral DBEs are ionized by free holes. Consequently, passivation of free holes should increase the efficiency of visible emission, perhaps to the point that annealed $\mathrm{ZnO}$ may be used as a viable alternate visible light phosphor.

\section{ASSOCIATED CONTENT}

\section{S Supporting Information}

The Supporting Information is available free of charge on the ACS Publications website at DOI: 10.1021/acs.jpcc.5b12094.

Full $\mathrm{ZnO}$ IR spectra, change in PL band shape during annealing, $\mathrm{MeOH}$ decomposition products at $300 \mathrm{~K}$, scavenger dosing at $300 \mathrm{~K}, \Delta \mathrm{IR}$ absorbance after $\mathrm{O}_{2}$ and $\mathrm{MeOH}$ exposure, fitting of PL decay during UV exposure, and PL band shape insensitivity to scanvengers (PDF)

\section{AUTHOR INFORMATION}

\section{Corresponding Author}

*E-mail everitt@phy.duke.edu (H.O.E.).

\section{Notes}

The authors declare no competing financial interest.

\section{ACKNOWLEDGMENTS}

The authors acknowledge the critical role John T. Yates played encouraging and supporting the pursuit this project. The authors also acknowledge helpful discussions with J. G. Simmons and J. V. Foreman. We acknowledge with thanks the support of this work by the Army Research Office under Grant 55748CH and the AES Corporation for a fellowship for Shiliang Ma through the AES Graduate Fellowship in the Energy Research Program at the University of Virginia.

\section{REFERENCES}

(1) Özgür, Ü.; Alivov, Y. I.; Liu, C.; Teke, A.; Reshchikov, M. A.; Doğan, S.; Avrutin, V.; Cho, S. J.; Morkoç, H. A Comprehensive Review of $\mathrm{ZnO}$ Materials and Devices. I. Appl. Phvs. 2005, 98, 041301.

(2) Choi, S.; Berhane, A. M.; Gentle, A.; Ton-That, C.; Phillips, M. R; Aharonovich, I. Electroluminescence from Localized Defects in Zinc Oxide: Toward Electrically Driven Single Photon Sources at Room Temperature. ACS Appl. Mater. Interfaces 2015, 7, 5619-5623.

(3) Foreman, J. V.; Everitt, H. O.; Yang, J.; Liu, J. Influence of Temperature and Photoexcitation Density on the Quantum Efficiency of Defect Emission in $\mathrm{ZnO}$ Powders. Appl. Phvs. Lett. 2007, 91, 011902.

(4) Dietz, R. E.; Kamimura, H.; Sturge, M. D.; Yariv, A. Electronic Structure of Copper Impurities in ZnO. Phys. Rev. 1963, 132, 15591569.

(5) Dingle, R. Luminescent Transitions Associated with Divalent Copper Impurities and the Green Emission from Semiconducting Zinc Oxide. Phvs. Rev. Lett. 1969, 23, 579-581.

(6) Garces, N. Y.; Wang, L.; Bai, L.; Giles, N. C.; Halliburton, L. E.; Cantwell, G. Role of Copper in the Green Luminescence from $\mathrm{ZnO}$ Crystals. Appl. Phys. Lett. 2002, 81, 622-624.

(7) Vanheusden, K.; Warren, W. L.; Seager, C. H.; Tallant, D. R.; Voigt, J. A.; Gnade, B. E. Mechanisms behind Green Photoluminescence in $\mathrm{ZnO}$ Phosphor Powders. I. Appl. Phvs. 1996, 79, 7983-7990.

(8) Vanheusden, K.; Seager, C. H.; Warren, W. L.; Tallant, D. R.; Voigt, J. A. Correlation between Photoluminescence and Oxygen Vacancies in ZnO Phosphors. Appl. Phys. Lett. 1996, 68, 403-405.

(9) Vanheusden, K.; Seager, C. H.; Warren, W. L.; Tallant, D. R.; Caruso, J.; Hampden-Smith, M. J.; Kodas, T. T. Green Photoluminescence Efficiency and Free-Carrier Density in $\mathrm{ZnO}$ Phosphor Powders Prepared by Spray Pyrolysis. L. Lumin. 1997, 75, 11-16.

(10) Studenikin, S. A.; Golego, N.; Cocivera, M. Fabrication of Green and Orange Photoluminescent, Undoped $\mathrm{ZnO}$ Films Using Spray Pyrolysis Fabrication of Green and Orange Photoluminescent, Undoped ZnO Films Using Spray Pyrolysis. I. Appl. Phvs. 1998, 84, 2287-2294.

(11) Leiter, F. H.; Alves, H. R.; Hofstaetter, A.; Hofmann, D. M.; Meyer, B. K. The Oxygen Vacancy as the Origin of a Green Emission in Undoped ZnO. Phvs. Status Solidi B 2001, 226, R4-R5.

(12) Janotti, A.; Van De Walle, C. G. Oxygen Vacancies in $\mathrm{ZnO}$. Appl. Phys. Lett. 2005, 87, 122102.

(13) Yang, X.; Du, G.; Wang, X.; Wang, J.; Liu, B.; Zhang, Y.; Liu, D.; Liu, D.; Ong, H. C.; Yang, S. Effect of Post-Thermal Annealing on Properties of $\mathrm{ZnO}$ Thin Film Grown on c-Al2O3 by Metal-Organic Chemical Vapor Deposition. L. Crust. Growth 2003, 252, 275-278.

(14) Kohan, A. F.; Ceder, G.; Morgan, D.; Van de Walle, C. G. FirstPrinciples Study of Native Point Defects in ZnO. Phvs. Rev. B: Condens. Matter Mater. Phys. 2000, 61, 15019-15027. 
(15) Egelhaaf, H.-J.; Oelkrug, D. Luminescence and Nonradiative Deactivation of Excited States Involving Oxygen Defect Centers in Polycrystalline ZnO. I. Cryst. Growth 1996, 161, 190-194.

(16) Guo, B.; Qiu, Z. R.; Wong, K. S. Intensity Dependence and Transient Dynamics of Donor-Acceptor Pair Recombination in $\mathrm{ZnO}$ Thin Films Grown on (001) Silicon. Appl. Phvs. Lett. 2003, 82, 22902292

(17) Knutsen, K. E.; Galeckas, A.; Zubiaga, A.; Tuomisto, F.; Farlow, G. C.; Svensson, B. G.; Kuznetsov, A. Y. Zinc Vacancy and Oxygen Interstitial in $\mathrm{ZnO}$ Revealed by Sequential Annealing and Electron Irradiation. Phys. Rev. B: Condens. Matter Mater. Phvs. 2012, 86, 121203.

(18) Ischenko, V.; Polarz, S.; Grote, D.; Stavarache, V.; Fink, K.; Driess, M. Zinc Oxide Nanoparticles with Defects. Adv. Funct. Mater. 2005, 15, 1945-1954.

(19) Korsunska, N. O.; Borkovska, L. V.; Bulakh, B. M.; Khomenkova, L. Y.; Kushnirenko, V. I.; Markevich, I. V. The Influence of Defect Drift in External Electric Field on Green Luminescence of $\mathrm{ZnO}$ Single Crystals. I. Lumin. 2003, 102, 733-736.

(20) Lin, B.; Fu, Z.; Jia, Y. Green Luminescent Center in Undoped Zinc Oxide Films Deposited on Silicon Substrates. Appl. Phvs. Lett. 2001, 79, 943-945.

(21) Stavale, F.; Nilius, N.; Freund, H. STM Luminescence Spectroscopy of Intrinsic Defects in $\mathrm{ZnO}(000 \overline{1})$ Thin Films. $\underline{\text { L.Phys. }}$ Chem. Lett. 2013, 4, 3972-3976.

(22) Ton-That, C.; Weston, L.; Phillips, M. R. Characteristics of Point Defects in the Green Luminescence from $\mathrm{Zn}$ - and O-Rich $\mathrm{ZnO}$. Phvs. Rev. B: Condens. Matter Mater. Phvs. 2012, 86, 115205.

(23) Choi, S.; Phillips, M. R.; Aharonovich, I.; Pornsuwan, S.; Cowie, B. C. C.; Ton-That, C. Photophysics of Point Defects in $\mathrm{ZnO}$ Nanoparticles. Adv. Opt. Mater. 2015, 3, 821-827.

(24) Weiher, R. Optical Properties of Free Electrons in $\mathrm{ZnO}$. Phys. Rev. 1966, 152, 736-739.

(25) Berger, T.; Sterrer, M.; Diwald, O.; Knözinger, E.; Panayotov, D.; Thompson, T. L.; Yates, J. T. Light-Induced Charge Separation in Anatase $\mathrm{TiO}_{2}$ Particles. I. Phvs. Chem. B 2005, 109, 6061-6068.

(26) Boccuzzi, F.; Chiorino, A.; Ghiotti, G.; Guglielminotti, E. Metal/ $\mathrm{n}$-Zinc Oxide Interaction: Effect of the Surrounding Atmosphere on IR Transparency. Langmuir 1989, 5, 66-70.

(27) Wolden, C. A.; Barnes, T. M.; Baxter, J. B.; Aydil, E. S. Infrared Detection of Hydrogen-Generated Free Carriers in Polycrystalline ZnO Thin Films. I. Appl. Phys. 2005, 97, 043522.

(28) McCluskey, M. D.; Jokela, S. J.; Zhuravlev, K. K.; Simpson, P. J.; Lynn, K. G. Infrared Spectroscopy of Hydrogen in ZnO. Appl. Phvs. Lett. 2002, 81, 3807-3809.

(29) Heiland, G.; Lüth, H. Multiphonon Infrared Absorption in Zinc Oxide. Solid State Commun. 1967, 5, 199-202.

(30) Lüth, H. Infrared Electroabsorption of Zinc Oxide in the MultiPhonon Region. Solid State Commun. 1969, 7, 585-588.

(31) Ashkenov, N.; Mbenkum, B. N.; Bundesmann, C.; Riede, V.; Lorenz, M.; Spemann, D.; Kaidashev, E. M.; Kasic, A.; Schubert, M.; Grundmann, M.; et al. Infrared Dielectric Functions and Phonon Modes of High-Quality ZnO Films. I. Appl. Phvs. 2003, 93, 126-133.

(32) Boccuzzi, F.; Morterra, C.; Scala, R.; Zecchina, A. Infrared Spectrum of Microcrystalline Zinc Oxide. Electronic and Vibrational Contributions under Different Temperature and Environmental Conditions. I. Chem. Soc. Faradav Trans. 2 1981, 77, 2059-2066.

(33) Boccuzzi, F.; Ghiotti, G.; Chiorino, A. Optical Properties of Microcrystalline Zinc Oxide. Influence of Defects Produced by Interaction with Carbon Monoxide. I. Chem. Soc., Faradav Trans. 2 1983, 79, 1779-1789.

(34) Göpel, W.; Lampe, U. Influence of Defects on the Electronic Structure of Zinc Oxide Surfaces. Phvs. Rev. B: Condens. Matter Mater. Phvs. 1980, 22, 6447-6462.

(35) Foreman, J. V.; Simmons, J. G.; Baughman, W. E.; Liu, J.; Everitt, H. O. Localized Excitons Mediate Defect Emission in $\mathrm{ZnO}$ Powders. I. Appl. Phys. 2013, 113, 133513.
(36) Basu, P.; Ballinger, T. H.; Yates, J. T., Jr. Wide Temperature Range IR Spectroscopy Cell for Studies of Adsorption and Desorption on High Area Solids. Rev. Sci. Instrum. 1988, 59, 1321-1327.

(37) Yates, Jr., J. T. Experimental Innovsations in Surface Science: A Guide to Practical Laboratory Methods and Instruments; Springer: New York, 1998.

(38) Panayotov, D. A.; Burrows, S. P.; Morris, J. R. Infrared Spectroscopic Studies of Conduction Band and Trapped Electrons in UV-Photoexcited, H-Atom N-Doped, and Thermally Reduced TiO 2. I. Phys. Chem. C 2012, 116, 4535-4544.

(39) Zhao, H.; Zhang, Q.; Weng, Y.-X. Deep Surface Trap Filling by Photoinduced Carriers and Interparticle Electron Transport Observed in $\mathrm{TiO}_{2}$ Nanocrystalline Film with Time-Resolved Visible and Mid-IR Transient Spectroscopies. L. Phvs. Chem. C 2007, 111, 3762-3769.

(40) Panayotov, D. A.; Yates, J. T., Jr. Depletion of Conduction Band Electrons in $\mathrm{TiO}_{2}$ by Water Chemisorption - IR Spectroscopic Studies of the Independence of $\mathrm{Ti}-\mathrm{OH}$ Frequencies on Electron Concentration. Chem. Phvs. Lett. 2005, 410, 11-17.

(41) Look, D. C. Recent Advances in $\mathrm{ZnO}$ Materials and Devices. Mater. Sci. Eng. B 2001, 80, 383-387.

(42) Erhart, P.; Albe, K.; Klein, A. First-Principles Study of Intrinsic Point Defects in $\mathrm{ZnO}$ : Role of Band Structure, Volume Relaxation, and Finite-Size Effects. Phvs. Rev. B: Condens. Matter Mater. Phvs. 2006, 73, 205203.

(43) Tomlins, G. W.; Routbort, J. L.; Mason, T. O. Zinc SelfDiffusion, Electrical Properties, and Defect Structure of Undoped, Single Crystal Zinc Oxide. I. Appl. Phvs. 2000, 87, 117-123.

(44) Janotti, A.; Van De Walle, C. G. Oxygen Vacancies in ZnO. Appl. Phvs. Lett. 2005, 87, 122102.

(45) Janotti, A.; Van de Walle, C. G. Fundamentals of Zinc Oxide as a Semiconductor. Rep. Prog. Phvs. 2009, 72, 126501.

(46) van Dijken, A.; Meulenkamp, E. A.; Vanmaekelbergh, D.; Meijerink, A. Influence of Adsorbed Oxygen on the Emission Properties of Nanocrystalline ZnO Particles. I. Phys. Chem. B 2000, $104,4355-4360$.

(47) van Dijken, A.; Meulenkamp, E. A.; Vanmaekelbergh, D.; Meijerink, A. The Kinetics of the Radiative and Nonradiative Processes in Nanocrystalline ZnO Particles upon Photoexcitation. I. Phys. Chem. $\underline{\underline{B}}$ 2000, 104, 1715-1723.

(48) Simmons, J. G., Jr.; Foreman, J. V.; Liu, J.; Everitt, H. O. The Dependence of $\mathrm{ZnO}$ Photoluminescence Efficiency on Excitation Conditions and Defect Densities. Appl. Phvs. Lett. 2013, 103, 201110.

(49) Van Dijken, A.; Meulenkamp, E. A.; Vanmaekelbergh, D.; Meijerink, A. Identification of the Transition Responsible for the Visible Emission in ZnO Using Quantum Size Effects. L. Lumin. 2000, 90, 123-128.

(50) Stroyuk, O. L.; Dzhagan, V. M.; Shvalagin, V. V.; Kuchmiy, S. Y. Size-Dependent Optical Properties of Colloidal ZnO Nanoparticles Charged by Photoexcitation. I. Phys. Chem. C 2010, 114, 220-225.

(51) Meyer, B. K.; Sann, J.; Lautenschläger, S.; Wagner, M. R.; Hoffmann, A. Ionized and Neutral Donor-Bound Excitons in $\mathrm{ZnO}$. Phvs. Rev. B: Condens. Matter Mater. Phys. 2007, 76, 184120.

(52) Meyer, B. K.; Alves, H.; Hofmann, D. M.; Kriegseis, W.; Forster, D.; Bertram, F.; Christen, J.; Hoffmann, A.; Straßburg, M.; Dworzak, M.; et al. Bound Exciton and Donor-acceptor Pair Recombinations in ZnO. Phvs. Status Solidi B 2004, 241, 231-260.

(53) Kodama, K.; Uchino, T. Thermally Activated below-Band-Gap Excitation behind Green Photoluminescence in ZnO. I. Appl. Phvs. 2012, 111, 093525. 